\title{
BMJ Open Wool-derived keratin dressings versus usual care dressings for treatment of slow healing venous leg ulceration: a randomised controlled trial (Keratin4VLU)
}

\author{
Andrew Jull (1) , ${ }^{1,2}$ Angela Wadham, ${ }^{2}$ Chris Bullen, ${ }^{2}$ Varsha Parag, ${ }^{2}$ \\ Carolina Weller (1) , ${ }^{3}$ Jill Waters ${ }^{4}$
}

To cite: Jull A, Wadham A, Bullen C, et al. Wool-derived keratin dressings versus usual care dressings for treatment of slow healing venous leg ulceration: a randomised controlled trial (Keratin4VLU). BMJ Open 2020;10:e036476. doi:10.1136/ bmjopen-2019-036476

- Prepublication history for this paper is available online. To view these files, please visit the journal online (http://dx.doi org/10.1136/bmjopen-2019036476).

Received 17 December 2019

Revised 26 March 2020

Accepted 04 June 2020

Check for updates

(c) Author(s) (or their employer(s)) 2020. Re-use permitted under CC BY-NC. No commercial re-use. See rights and permissions. Published by BMJ.

${ }^{1}$ School of Nursing, University of Auckland, Auckland, Auckland, New Zealand

${ }^{2}$ National Institute for Health Innovation, University of Auckland, Auckland, New Zealand

${ }^{3}$ School of Nursing and Midwifery, Monash University, Melbourne, Victoria, Australia

${ }^{4}$ Hope Foundation for Research on Ageing, Auckland, New Zealand

Correspondence to Professor Andrew Jull; a.jull@auckland.ac.nz

\section{ABSTRACT}

Objective To determine the effect of a keratin dressing for treating slow-to-heal venous leg ulcers (VLU) on VLU healing.

Design Pragmatic parallel group randomised controlled trial.

Setting Community-dwelling participants.

Participants People aged 18 or more years with VLU (either present for more than 26 weeks or ulcer area larger than $5 \mathrm{~cm}^{2}$ or both).

Intervention Wool-derived keratin dressing or usual care formulary of non-medicated dressings, on a background treatment with compression.

Primary and secondary outcome measures Healing at 24 weeks based on blinded assessment of ulcer photographs. Other outcomes included time to complete healing, change in ulcer area to 24 weeks, change in health-related quality of life and incidence of adverse events.

Results We screened 1068 patients with VLU and randomised 143 participants (51.1\% of target recruitment), 71 to the keratin dressing group and 72 to the usual care group.The mean age was 66.1 years (SD 15.9) and 53 participants $(37.1 \%)$ were women. There were no significant differences between the groups on the primary outcome (risk difference $-6.4 \%, 95 \% \mathrm{Cl}-22.5 \%$ to $9.7 \%$ ), change in ulcer area $\left(-1.9 \mathrm{~cm}^{2}, 95 \% \mathrm{Cl}-16.5\right.$ to 12.8 $\mathrm{cm}^{2}$ ), time to complete healing (HR $0.80,95 \% \mathrm{Cl} 0.52$ to 1.23) or the incidence of adverse events (incidence rate ratio $1.19,95 \% \mathrm{Cl} 0.89$ to 1.59 ) in the intention-to-treat analyses. However, the direction of effect on the primary outcome was reversed in a per protocol analysis specified a priori (risk difference $6.2 \%, 95 \% \mathrm{Cl}-12.4 \%$ to $24.9 \%$ ). Conclusion The effect of adding a keratin dressing to the treatment regimen for prognostically slow-to-heal VLU remains unclear.

Trial registration number NCT02896725

\section{INTRODUCTION}

Keratins are filament-forming proteins produced by activated keratinocytes that migrate from the wound margins in healing
Strengths and limitations of this study

- This study was a pragmatic trial conducted in community settings in ways that replicate real-world venous leg ulcer care.

- We used a validated prognostic tool to identify patients likely to be slow-to-heal with 24 weeks compression.

- About two-thirds of the 1068 screened patients were ineligible, a greater proportion than we anticipated in our sample size calculation.

- We were unable to recruit our target sample size within the available funding time frame.

wounds. ${ }^{1}$ Keratins 6,16 and 17 are required for migration, ${ }^{2}$ but when compared with healing venous leg ulcers (VLU), biopsies from slow healing VLU show keratin to be downregulated. ${ }^{3}$ Introducing exogenous wool-derived keratins promotes both in vitro keratinocyte migration and in vivo porcine healing. ${ }^{45}$ Endogenous keratin was expressed earlier in wounds treated with wool-derived keratin, suggesting the exogenous keratin was inducing endogenous expression, not simply donating exogenous keratin. ${ }^{5}$

VLU can be categorised into 'normal healing' and two levels of 'slow healing' using the Margolis index. ${ }^{6}$ This prognostic index categorises patients' likelihood of healing based on the area and duration of VLU. Trials that recruit only those participants with slow healing VLU report delayed time-to-healing (eg, median time-to-healing 245-338 days) ${ }^{78}$ compared with trials that recruit participants with any type of VLU (eg, median time-tohealing 69-98 days). ${ }^{9-11}$

We aimed to investigate whether using a wool-derived keratin dressing, in addition to 
compression, increased healing in patients with a slowhealing VLU.

\section{METHODS}

The trial design has been detailed elsewhere. ${ }^{12}$ Briefly, Keratin4VLU was a pragmatic parallel group single blinded randomised controlled trial conducted from March 2017 to February 2019. We sought to recruit community-dwelling participants from patients receiving VLU management in five study centres based within community nursing services in New Zealand (Auckland, South Auckland, Waikato, Christchurch or Dunedin districts from March 2017) and two study centres in Melbourne, Australia (Caulfield Hospital and Austin Health, from April 2018 to bolster recruitment).

\section{Participant recruitment}

Patients were eligible if they were aged 18 years or older with a VLU, were able to tolerate compression therapy, could provide written informed consent, and the VLU had been present for more than 26 weeks and/or was larger than $5 \mathrm{~cm}^{2}$ at randomisation. Patients met the case definition for venous leg ulceration if they had an incident or prevalent ulcer, where the presenting aetiology was venous (irregular shape, shallow and moist, with associated haemosiderin pigmentation, ankle flare, or lipodermatosclerosis), the Ankle Brachial Index (ABI) was greater than 0.7 and other causes had been ruled out. We had initially used an ABI of greater than 0.8, but relaxed this criterion to try and increase recruitment.

\section{Randomisation, allocation concealment and blinding}

Randomisation was 1:1 allocation stratified by study centre and the Margolis index score, which was used to assess the likelihood of participants' healing within 24 weeks when treated with compression. This prognostic index was based on the presence of one factor from either ulcer duration (ulcer present for 26 or more weeks) or ulcer area (ulcer size greater than $5 \mathrm{~cm}^{2}$ ) or the presence of both factors. The study statistician created a computer-generated random sequence (using randomly varying block sizes of 2 and 4) in separate lists for each study centre and prognostic level. These lists were loaded into Research Electronic Data Capture (REDCap) to be accessed by the research nurses using a computer tablet, with allocation only revealed after participant details were recorded. Trained research nurses screened and registered potential participants at each site. Eligible participants were consented on registration and were randomised after a 2-week run-in period. The participants and research nurses were aware of the treatment allocation, but the coder, adverse event and outcome adjudicators were blinded to allocation.

\section{Interventions}

Following the 2-week run-in phase to ensure compliance with compression and to remove early healers, patients were reassessed for eligibility at week 0 before being randomised. The intervention keratin dressing (Keramatrix) was manufactured and supplied to the trial by Keraplast Technologies, New Zealand. The keratin dressing was moistened using sterile saline and applied directly to the VLU. Where the potential for maceration was a concern, the dressing was cut to the size of the VLU before application. A secondary dressing could be used for extra absorbency. The dressing was changed at each visit with a target frequency of weekly visits (modified as indicated by patient need).

The control treatment was a non-medicated dressing selected on the basis of clinician and/or participant preference from the formulary of dressings available at each study centre. These dressings included hydrogels, alginates, hydrofibres, polyurethane foams and siliconimpregnated dressings. Other absorbent dressings (eg, combine dressings) could also be used as a secondary dressing.

All participants were treated with compression, with the chosen system being guided by clinician and/or participant preference and system availability at each study centre. Where the participant developed a local infection

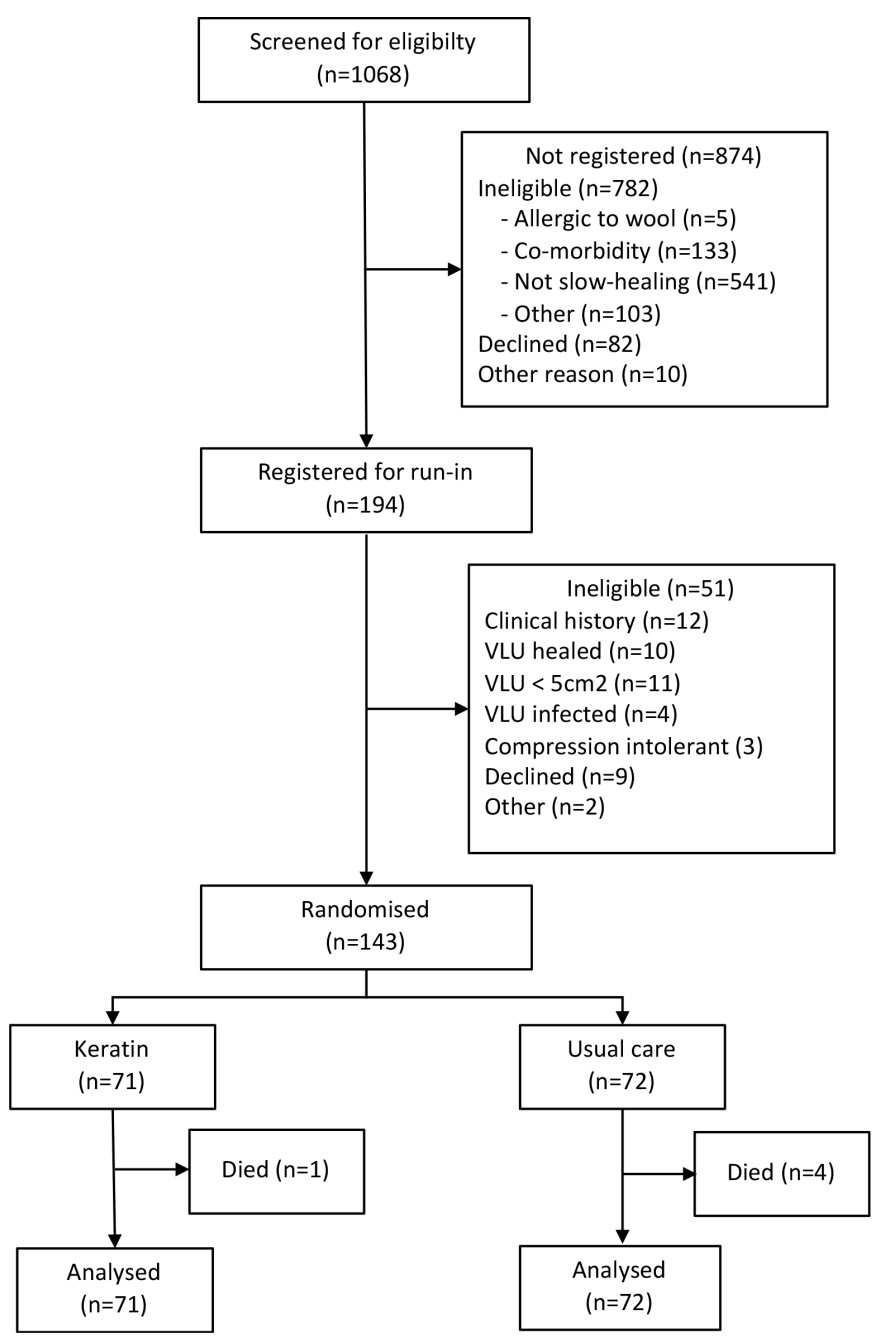

Figure 1 Participant flow diagram. All screened participants had venous leg ulcers. 
Table 1 Baseline characteristics for randomised trial participants (number and percentage unless otherwise stated)

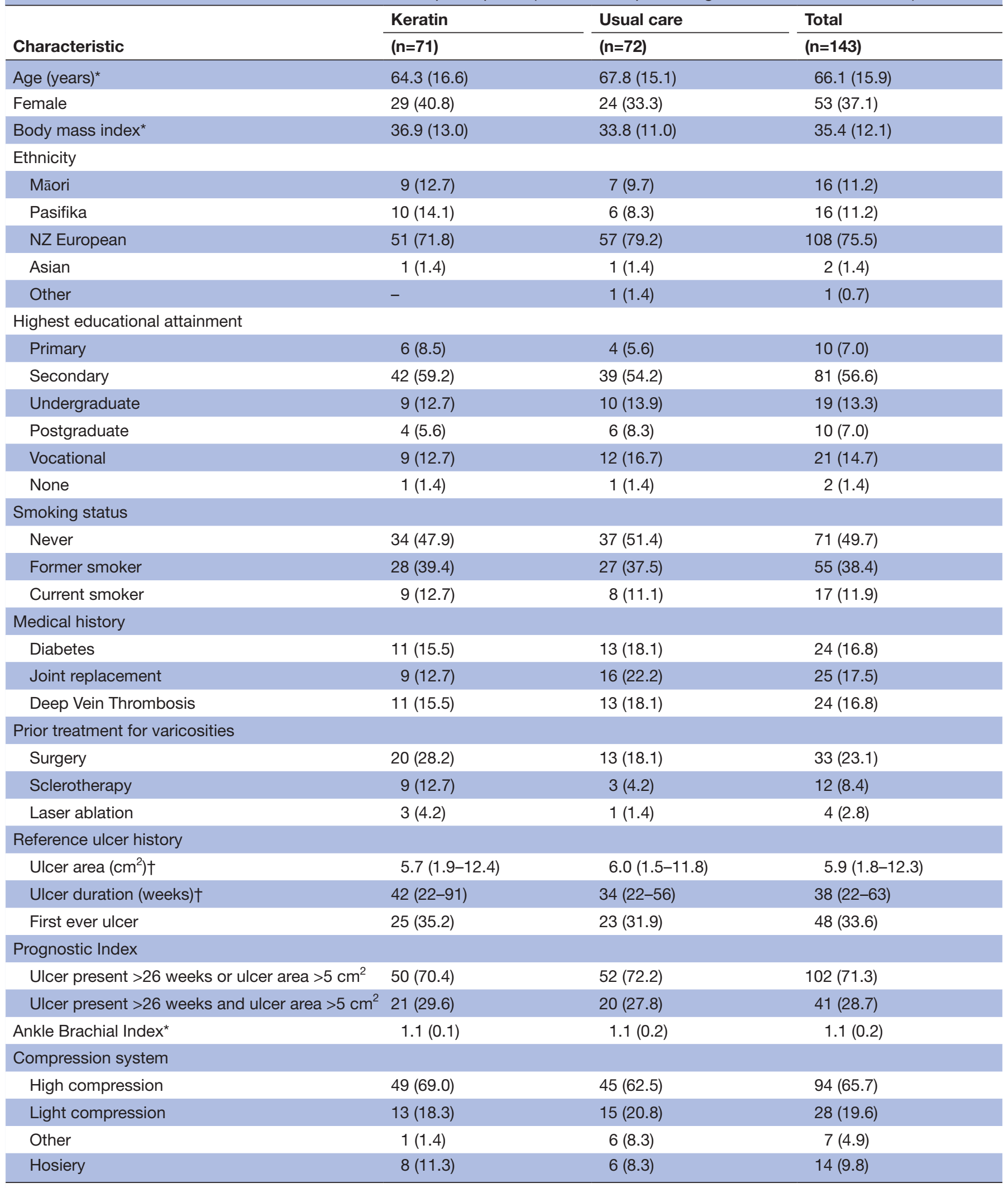

${ }^{*}$ Mean (SD).

†Median (IQR).

NZ, New Zealand. 
Table 2 Reasons by group participants were excluded for the per-protocol analysis of healing at 24 weeks (number and percentage)

\begin{tabular}{lll}
\hline Reason & Keratin $(\mathbf{n = 2 4 )}$ Usual care (n=6) & $2(33.3)$ \\
\hline Deceased before 24 weeks visit & - & \\
\hline Withdrew & $3(12.5)$ & - \\
\hline Increasing pain/discomfort & $2(8.3)$ & - \\
\hline Medical advice (infection) & $5(20.8)$ & $1(16.7)$ \\
\hline Participant decision (infection) & $3(12.5)$ & - \\
\hline Participant decision (not progressing) & $2(8.3)$ & $2(33.3)$ \\
\hline Stopped compression & $1(4.2)$ & - \\
\hline Reaction to secondary dressing (Mepilex) & $7(29.2)$ & - \\
\hline Participant decision (no reason given) & $1(4.2)$ & $1(16.7)$ \\
\hline Largest ulcer not chosen as reference & & \\
\hline
\end{tabular}

related to the VLU, ${ }^{13}$ the allocated study treatment was temporarily halted and a medicated dressing was used as an alternate for a maximum of 2 weeks. The medicated dressing was selected from the formulary of medicated dressings available at each study centre, including silver, iodine or honey-impregnated dressings. For unresponsive or spreading infections, ${ }^{13}$ the participant was referred to a primary care practitioner for assessment and prescription of antibiotics. Once infection was resolved, the allocated treatment was recommenced. Each infection was considered an adverse event.

\section{Measurements}

The primary outcome was the number of participants to have a healed reference ulcer (the largest baseline VLU where more than one ulcer was present) at 24 weeks as adjudicated by the blinded assessment of ulcer photographs. Healing was defined as complete epithelialisation of the reference ulcer with absence of scab.

The secondary outcomes were time to complete healing, agreement between the blinded and unblinded assessors on healing assessments, estimated change in

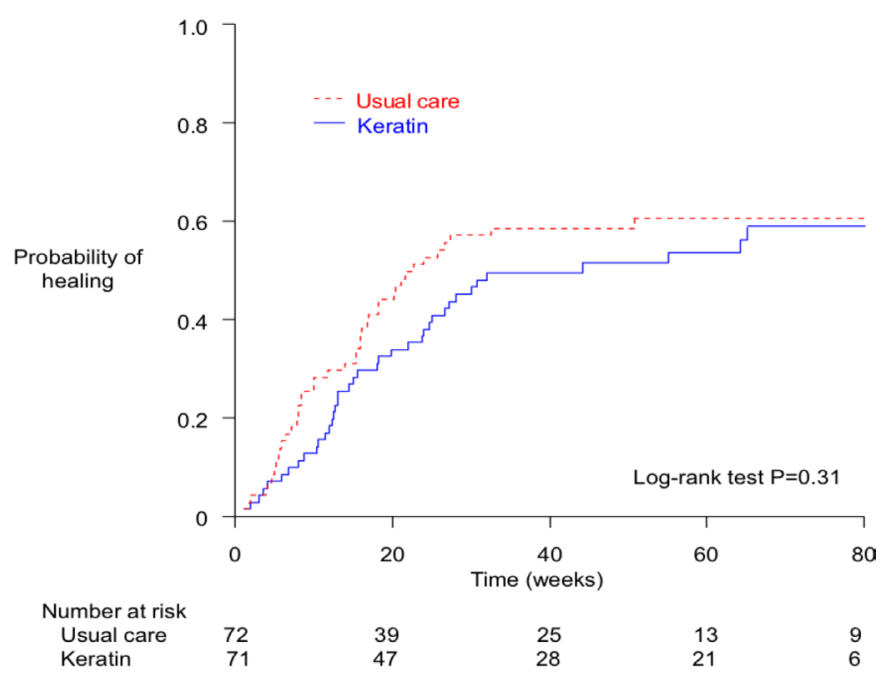

Figure 2 Kaplan-Meier plot for time to complete healing of the reference ulcer by trial treatment group. ulcer area, change in health-related quality of life and incidence of adverse events. When notified by the patient or district nurse of ulcer healing, the research nurse visited either before or after the 24 weeks endpoint to photograph the ulcer. The status of the ulcer (healed/ unhealed) was determined by blinded adjudication as per the primary outcome. Change in ulcer area was estimated using baseline and 24 weeks endpoint calculations for the area of an ellipse based on measurements made by the research nurse of the maximum length and width (at $90^{\circ}$ to maximum length) of the reference ulcer using either a disposable tape measure or measurement off an acetate tracing. The area of an ellipse is highly correlated with actual area. ${ }^{14}$ Change in health-related quality of life was assessed using RAND-36, EuroQol 5D3 Level (EQ5D3L) and the Charing Cross Venous Ulcer Questionnaire (CXVUQ) with the instruments completed at baseline and 24 weeks. Adverse events were defined as any untoward medical event regardless of causal relationship with the treatment and were coded using the International Statistical Classification of Diseases Related Health Problems version 10 (ICD-10) or study specific ulcer codes. Adverse event causality was assigned by a blinded reviewer. Adverse events for each participant were captured up to the point of healing or for the duration of trial if the participant was unhealed. We also noted in the trial protocol that cost-effectiveness modelling would be conducted, but given the lack of a consistent and clear effect, we did not pursue such modelling.

\section{Sample size}

We estimated that a total sample size of 252 would be sufficient to show a $20 \%$ increase in the percentage of participants with a completely healed reference ulcer at 24 weeks, assuming an alpha of 0.05 , a control group healing rate of $38 \%$ and $90 \%$ power. This absolute increase in healing was smaller than the $36 \%$ treatment effect suggested in the only previous controlled study. ${ }^{15}$ We allowed for a $10 \%$ lost to follow-up, and thus believed 140 in each group would be required if our assumptions were correct (total $n=280$ ). 
Table 3 Continuous outcomes (all mean differences adjusted for baseline values)

\begin{tabular}{|c|c|c|c|c|c|}
\hline \multirow{2}{*}{ Outcome } & \multicolumn{2}{|l|}{ Keratin } & \multicolumn{2}{|l|}{ Usual care } & \multirow[b]{2}{*}{$\begin{array}{l}\text { Mean difference in change at } \\
24 \text { weeks }(95 \% \mathrm{Cl})^{\star}\end{array}$} \\
\hline & $\begin{array}{l}\text { Baseline } \\
\text { (SD) }\end{array}$ & $\begin{array}{l}\text { Change at } 24 \\
\text { weeks (SE) }\end{array}$ & $\begin{array}{l}\text { Baseline } \\
\text { (SD) }\end{array}$ & $\begin{array}{l}\text { Change at } 24 \\
\text { weeks (SE) }\end{array}$ & \\
\hline Change in ulcer area $\left(\mathrm{cm}^{2}\right)$ & $12.6(20.0)$ & $-0.4(5.2)$ & $10.5(14.4)$ & $-2.3(5.2)$ & $-1.9(-16.5$ to 12.8$)$ \\
\hline Sensitivity analysis $†$ & $11.0(16.7)$ & $-5.6(0.9)$ & $10.4(14.5)$ & $-7.2(0.9)$ & $-1.6(-4.0$ to 0.8$)$ \\
\hline Role physical & $44.9(41.8)$ & $4.4(4.5)$ & $38.9(42.7)$ & $7.9(4.4)$ & $-3.5(-16.0$ to 9.0$)$ \\
\hline Bodily pain & $51.9(28.3)$ & $4.3(2.7)$ & $48.7(24.4)$ & $3.9(2.7)$ & $0.4(-7.2$ to 7.9$)$ \\
\hline General health & $58.7(21.7)$ & $3.3(1.8)$ & $56.4(20.4)$ & $2.7(1.8)$ & $0.6(-4.4$ to 5.7$)$ \\
\hline Vitality & $54.8(23.7)$ & $4.1(2.1)$ & $51.5(19.9)$ & $-0.5(2.0)$ & $4.6(-1.1$ to 10.4$)$ \\
\hline $\begin{array}{l}\text { Physical Component Summary } \\
\text { score }\end{array}$ & $36.2(10.4)$ & $0.8(1.1)$ & $34.2(9.7)$ & $1.2(1.1)$ & $-0.4(-3.4$ to 2.6$)$ \\
\hline $\begin{array}{l}\text { Mental Component Summary } \\
\text { score }\end{array}$ & $46.0(13.0)$ & $3.3(1.2)$ & $45.0(11.2)$ & $1.5(1.2)$ & $1.8(-1.5$ to 5.1$)$ \\
\hline \multicolumn{6}{|c|}{ Change in EuroQol-5D-3 Level (higher scores better, positive value for change score indicates gain) } \\
\hline Health state & $67.3(17.7)$ & $6.4(1.7)$ & $64.4(19.7)$ & $1.1(1.7)$ & $5.2 \ddagger(0.5$ to 9.9$) \S$ \\
\hline Utility index & $0.6(0.3)$ & $0.05(0.03)$ & $0.6(0.2)$ & $0.07(0.03)$ & $-0.01 \ddagger(-0.10$ to 0.07$)$ \\
\hline \multicolumn{6}{|c|}{ Change in Charing Cross Venous Ulcer Questionnaire score (lower scores better, negative value for change score indicates gain) } \\
\hline Social function & $43.8(19.2)$ & $-1.8(1.5)$ & $42.9(16.4)$ & $-5.5(1.5)$ & $-3.7(-7.9$ to 0.5$)$ \\
\hline
\end{tabular}

*Negative value indicates greater change in the usual care group. †Four outlier values for ulcer area greater than $80 \mathrm{~cm}^{2}$ removed. $\ddagger$ Discrepancies due to rounding.

$\S \mathrm{P}=0.029$.

१P $=0.006$.

${ }^{* *} P=0.024$.

CXVUQ, Charing Cross Venous Ulcer Questionnaire.

\section{Statistics}

Data analyses were specified a priori in a statistical analysis plan (available on request). All analyses, excepting quality-of-life analyses, were first carried out using an intention-to-treat approach in SAS V.9.4 (SAS). Sensitivity analyses were undertaken on the primary outcome to determine the effect of protocol compliance and lost to follow-up. Agreement between research nurses and adjudicators was assessed using kappa. $\mathrm{X}^{2}$ tests, relative risks and risk differences (with $95 \%$ CIs) were calculated for binary outcomes in the first instance, with subsequent multiple logistic regression analysis conducted to adjust for stratification factors (study centre and prognostic index). For categorical healing outcomes, participants lost to follow-up were presumed to have an ulcer that remained unhealed, whereas for change in ulcer area baseline values were carried forward for missing data. Time-to-event data were analysed using a Kaplan-Meier plot and log-rank test, and Cox regression to adjust for stratification factors and censoring. Change in ulcer area and health-related quality of life outcomes (with $95 \%$ CIs) were analysed using multiple linear regression and adjusted for baseline value. Only participants that had paired baseline and endpoint data were included in the health-related quality of life analyses. Adverse events were analysed using incidence rate ratios (IRRs).

\section{Patient and public involvement}

We did not involve patients in the design or conduct of the trial. We surveyed 139 participants about their trial experience after their follow-up was completed. Although we did not expressly assess burden, $89.5 \%$ either strongly 
Table 4 Incidence of AEs by type of event and causal relationship

\begin{tabular}{|c|c|c|c|}
\hline Type of AE & $\begin{array}{l}\text { Keratin } \\
\mathbf{N} \text { of events }\end{array}$ & $\begin{array}{l}\text { Usual care } \\
\mathbf{N} \text { of events }\end{array}$ & $\begin{array}{l}\text { Incidence rate ratio } \\
(95 \% \mathrm{Cl})\end{array}$ \\
\hline Incidence of all AEs & 109 & 79 & 1.19 (0.89 to 1.59$)$ \\
\hline Probably related AEs & - & - & - \\
\hline Possibly related AEs & 4 & - & - \\
\hline $\begin{array}{l}\text { Probably related ulcer- } \\
\text { specific AEs }\end{array}$ & 4 & - & - \\
\hline $\begin{array}{l}\text { Possibly related ulcer- } \\
\text { specific AEs }\end{array}$ & 20 & 7 & - \\
\hline
\end{tabular}

\begin{tabular}{|c|c|c|c|c|c|c|}
\hline & \multicolumn{3}{|l|}{ Keratin } & \multicolumn{3}{|l|}{ Usual care } \\
\hline & $\begin{array}{l}\mathbf{N} \text { of events } \\
\text { (people) }\end{array}$ & $\begin{array}{l}\text { Probably } \\
\text { related }\end{array}$ & $\begin{array}{l}\text { Possibly } \\
\text { related }\end{array}$ & $\begin{array}{l}\mathbf{N} \text { of events } \\
\text { (people) }\end{array}$ & $\begin{array}{l}\text { Probably } \\
\text { related }\end{array}$ & $\begin{array}{l}\text { Possibly } \\
\text { related }\end{array}$ \\
\hline Accident & $1(1)$ & - & - & $2(2)$ & - & - \\
\hline Cancer & $3(3)$ & - & - & $1(1)$ & - & - \\
\hline Genitourinary & $1(1)$ & - & - & $4(2)$ & - & - \\
\hline Musculoskeletal & $2(2)$ & - & - & - & - & - \\
\hline Respiratory & $4(4)$ & - & - & $2(2)$ & - & - \\
\hline Skin and subcutaneous & $12(9)$ & - & 2 & $8(7)$ & - & - \\
\hline Debridement & $2(1)$ & - & - & - & - & - \\
\hline Pain & $4(4)$ & 2 & 1 & - & - & - \\
\hline Harm from dressing removal & $1(1)$ & 1 & - & - & - & - \\
\hline Maceration & $1(1)$ & - & 1 & $1(1)$ & - & 1 \\
\hline Increased erythema & $2(2)$ & - & 1 & $1(1)$ & - & - \\
\hline Increased oedema & $1(1)$ & - & - & $1(1)$ & - & - \\
\hline Increased venous dermatitis & $1(1)$ & - & - & $1(1)$ & - & 1 \\
\hline Increased venous eczema & $1(1)$ & - & - & - & - & - \\
\hline
\end{tabular}

*Includes serious AEs.

AEs, adverse events; ICD-10, International Statistical Classification of Diseases version 10.

agreed or agreed with the statement 'I would participate in another trial given a chance' with $6.7 \%$ neither agreeing nor disagreeing with the statement. Participants were also asked about agreement with the statement 'I would recommend being part of trial to other people';
89.8\% either strongly agreed or agreed and a further $10.2 \%$ neither agreed nor disagreed. We have included in the dissemination plan sending a lay summary of the results to participants via an email or posted letter, once the results are published in an open access journal. The 
communication will include a link to the open access publication.

\section{RESULTS}

We screened 1068 patients with VLU (figure 1). Overall, 194 could be registered to start the run-in phase, but 51 did not progress to randomisation, mostly because they healed, had an ulcer smaller than the specified area at randomisation, became infected or declined participation. We randomised 143 participants $(51.1 \%$ of target recruitment), 71 to the keratin dressing group and 72 to the usual care group, all in New Zealand. While we screened 70 patients in the Australian centres, no patients met the inclusion criteria. Five participants died, two before we could obtain information on the primary endpoint and both of whom were in the usual care group.

The groups were similar at baseline except for history of joint replacement, prior treatment for varicosities and duration of the reference ulcerin weeks (table 1). The first two variables were considered to act against the usual care group and therefore were not thought to require adjustment. The last variable was highly correlated with the Margolis index, which was already included in the analyses adjusted for stratification factors and thus the imbalance would have been controlled in those analyses. Overall, the mean age was 66.1 years (SD 15.9) while 53 participants $(37.1 \%)$ were women. Most participants were New Zealand European $(108,75.5 \%)$ with equal numbers of Māori $(16,11.2 \%)$ and Pasifika participants $(16,11.2 \%)$. Most participants were using high compression $(94,65.7 \%)$ or hosiery $(14,9.8 \%)$.

There was no significant difference between the groups on the primary outcome. Twenty-seven participants $(38.0 \%)$ in the keratin group healed by 24 weeks compared with 32 participants $(44.4 \%)$ in the usual care group (risk difference $-6.4 \%, 95 \%$ CI $-22.5 \%$ to $9.7 \%$ ). The relative risk (RR) of healing (RR $0.86,95 \mathrm{CI} \% 0.58$ to 1.27) was little different when adjusted for stratification factors (RR 0.91, 95\% CI 0.64 to 1.64). The direction of effect was reversed in the per protocol analysis that was specified a priori with a greater percentage of participants healed in the keratin group than the control group (30 participants were removed from the analysis for major protocol violations, death and withdrawal from treatment, 24 in the keratin group and six in the usual care group, table 2). Twenty-five (53.2\%) of the remaining 47 participants healed in the keratin group compared with $31(47.0 \%)$ of the remaining 66 participants in the usual care group (risk difference $6.2 \%, 95 \%$ CI $-12.4 \%$ to $24.9 \%$ ). The research nurses and blinded adjudicators agreed on $57 / 60$ assessments of healing and 77/79 assessments of non-healing at 24 weeks (kappa $0.93,95 \%$ CI 0.86 to 0.99$)$.

The change in estimated ulcer area at 24 weeks also did not differ significantly between the groups on either the main analysis (table 3), or in the sensitivity analysis (four outliers were removed, three in the keratin group and one in the usual care group). Participants were followed up until trial ended or the first healing event. There was no significant difference in time to complete healing with 39 participants $(54.9 \%)$ healing in the keratin group and 42 participant $(58.3 \%)$ healing in the usual care group (figure 2). Median time to healing was 309 days in the keratin group and 159 days in the usual care group (HR $0.80,95 \%$ CI 0.52 to 1.23$)$.

There were no significant differences on the change to health-related quality of life scores $(n=138)$ as measured by RAND-36 (table 3). However, the groups were significantly different on overall health state as measured by EQ5D-3L, with participants in the keratin group having greater gain than participants in the usual care group. Conversely, participants in the usual care group had significantly greater gains overall and for cosmetic appearance of the ulcerated leg, as measured by the disease-specific CXVUQ.

One hundred and nine adverse events in 45 participants were reported in the keratin group and 79 adverse events in 37 participants were reported in the usual care group, most of which were ulcer-specific events (table 4). There were no significant differences in the incidence of any adverse event (IRR $1.19,95 \%$ CI 0.89 to 1.59 ), adverse events coded using ICD-10 (IRR 1.05, 95\% CI 0.64 to 1.59$)$ or ulcer-specific adverse events $(1.27,95 \%$ CI 0.89 to 1.81 ). There were 17 serious adverse events in nine participants in the keratin group and 21 serious adverse events in 13 participants in the usual care group (table 5). There was no significant difference between the groups in the incidence of serious adverse events and no serious adverse event was probably or possibly related to treatment IRR $(0.70,95 \%$ CI 0.37 to 1.33$)$.

\section{DISCUSSION}

Our trial was the first randomised trial of wool-derived keratin for treating VLU. We were unable to recruit

Table 5 Incidence of serious adverse events by type of event

\begin{tabular}{lccc}
\hline Type of serious adverse event & $\begin{array}{l}\text { Keratin } \\
\text { N of events (people) }\end{array}$ & $\begin{array}{l}\text { Usual care } \\
\text { N of events (people) }\end{array}$ & $\begin{array}{l}\text { Incidence rate ratio } \\
(\mathbf{9 5 \%} \text { Cl) }\end{array}$ \\
\hline Death & $1(1)$ & $4(4)$ & - \\
Hospitalisation & $14(8)$ & $15(8)$ & - \\
Other significant event & $2(2)$ & $2(2)$ & - \\
Total & $17(9)$ & $21(13)$ & $0.70(0.37$ to 1.33$)$ \\
\hline
\end{tabular}


sufficient participants to meet our original objectives as the pool of slow-to-heal VLU seems to have reduced compared with our earlier trials. As a result, the treatment effect in slow-to-heal VLU remains unclear, with the proportion healed slightly lower in the keratin group on an intention to treat analysis, but slightly higher than in the control group on a per-protocol analysis. None of these effects were significantly different and it seems reasonable to assert, despite the lower than anticipated sample size, that the substantial increase in healing found in previous studies is unlikely.

A case series of seven patients with refractory VLU and a Margolis index of two (indicating an expected healing rate of $13 \%$ after 24 weeks treatment with compression) found five of the patients $(71.4 \%)$ healed by 12 weeks when treated with either a keratin-based hydrogel or foam. ${ }^{16}$ The authors suggested that these slow-to-heal VLU were an ideal target with which to evaluate the dressing in a randomised trial. However, in our trial up to 24 weeks exposure to treatment with keratin did not improve healing rates over usual care $(9.5 \%$ vs $10.0 \%$ respectively) in participants with a Margolis index equal to that in the case series. A non-randomised controlled trial of 55 patients with VLU, 31 in the keratin dressing group and 24 in the control group, reported a $36.3 \%$ absolute increase in healing after up to 16 weeks treatment with keratin. ${ }^{15}$ There is limited information available about this trial, making it difficult to determine why our findings are so different. However, the most obvious explanations are that confounding associated with nonrandom allocation and random error associated with a smaller sample size could have influenced the results in this earlier trial.

Our findings are similar to that of a within-participant randomised trial of 26 skin graft donor sites that found no overall healing effect for a keratin dressing. ${ }^{17}$ However, an age-related effect was suggested as there was a 5.3\% absolute difference in healing in favour of keratin dressings in the 11 participants aged older than 50 years. About $84 \%$ (120) of participants in our trial were aged over 50 years and we did not observe such an age-related effect on the intention to treat analysis, although we did find a $6 \%$ increase in healing after excluding participants for the per-protocol analysis. Thus, it is unclear whether an age-related effect does exist.

We found no between-group differences for the incidence of adverse events, although the reporting of adverse events was more frequent in exposed arm as would be expected in a trial with limited blinding. We did observe that a considerable number of participants developed an infection requiring treatment with antibiotics in our trial, although with similar numbers in both groups. This rate was lower than the $36.6 \%$ observed in a case series of keratin-treated chronic wounds, ${ }^{18}$ but similar to that of other pragmatic trials (infection rates of $20.2 \%,{ }^{19} 22.4 \%,{ }^{7}$ and $19.5 \%{ }^{20}$ respectively). Diagnosis of infection in VLU is difficult and these trials used clinical measures of infection. However, the rates do not differ from another trial that used biopsy confirmation of infection and found $19.9 \%$ of 614 screened patients had an infected VLU. ${ }^{21}$

Our trial had the strengths of randomisation, strong allocation concealment, blinded adjudication of the primary outcome and near complete follow-up. Apart from the lower than anticipated sample size, our trial is subject to three other possible limitations. First, we used a prognostic index to identify slow-to-heal VLU rather than await a population that had not responded to usual care. Our approach was dictated by the anticipated difficulty in recruiting such a refractory population. However, the 24-week healing rates for the two strata of slow-to-heal VLU (53.9\% and 9.8\%) were lower than the actual healing rates observed in the cohort of patients from which the Margolis index was originally derived $(65 \%$ and $13 \%) .{ }^{6}$ Further, the overall percentage healed at 24 weeks in our trial $(41.3 \%)$ was similar to that estimated from the survival curve of a trial that also recruited hard-to-heal participants (approximately $35 \%-37 \%) .{ }^{8}$ We used number healed at 24 weeks as the primary outcome. Second, we recognise it is more generally desirable to report time to event as a primary outcome in VLU trials. However, we did not have the necessary inputs for time to complete healing to be used in the sample size calculation and we did include time-to-complete healing as a secondary outcome. Further, the Food and Drug Administration industry guidance on outcomes for ulcer trials includes incidence of complete healing as a clinically meaningful outcome for ulcer trials. ${ }^{22}$ Third, the Early Venous Reflux Ablation (EVRA) trial demonstrating the effect of surgery on VLU healing was published towards the end of our trial raising the question of whether there was balance on participants offered venous surgery during this trial. Review of the line listings for all hospitalisations reported as serious adverse events showed no participants had any venous surgery during the trial.

Our efforts to increase participation by relaxing an inclusion criterion and adding two study centres to the trial did not result in any additional participants. One approach we explored but decided against changing was the length of the run-in period. While a short run-in is necessary to establish tolerance of compression for incident patients, we have limited that to 1 week in our previous trials. ${ }^{11} 2023$ For the current trial, we used 2 weeks in this trial following a reviewer suggestion to remove early healers. This decision led to 21 otherwise eligible participants being lost prior to randomisation because their ulcer size decreased sufficiently during the run-in period to render them ineligible. It also did not stop some participants from healing very soon after commencing treatment, which can be observed in any VLU trial reporting a survival curve. While the extra participants would not have been sufficient for us to meet our proposed sample size, the salutary lesson is that as many barriers as possible need to be removed to enhance participation and that includes the duration of run-in phases. 


\section{CONCLUSION}

The effect of adding a keratin dressing to the treatment regimen for prognostically slow-to-heal VLU remains unclear.

Twitter Andrew Jull @1CommonReader, Chris Bullen @DrChrisBullen and Carolina Weller@CarolinaDWeller

Acknowledgements The authors wish to thank the site investigators (Jodi Botting, Sue Fenwick, Trish Johns, Julie Betts, Maria Schollum, Cathy Hammond, Emil Schmidt) and the Research Nurses (Kate Wotton, Janet Patterson, Irene Cronje,Desley Rosevear, Bronwen Meredith,Sian Ellis, Sue Lee, Rosemary McGinnes, Louise Turnour) for their support of the 4VLU research collaboration. The authors wish to acknowledge the contribution of the members of the 4VLU Data Safety Monitoring Board, Professor Thomas Lumley, Associate Professor Joanne Barnes and Vanessa Selak. We thank the following National Institute for Health Innovation staff for their work operationalising the trial: Michelle Jenkins, John Faatui and Terry Holloway (data management), Kate Hudson and Sarah Douglas (administration). We thank Nick Kearns and Ben Pearson (contracts) from Auckland Uniservices. Finally, we thank the participants and their families and whanau for contributing their experience and data for the benefit of others. This trial would not have been possible without their altruism.

Contributors AJ conceived the study and is the guarantor. AJ, CB, VP, and JW designed the trial and obtained funding for the trial. CW led the Australian study centres. AJ (chair), AW, CB, VP, and JW developed the trial protocol, developed or reviewed study materials (Trial Protocol Manual of Procedures, Case Record Forms), and were members of the trial steering committee. CB reviewed all adverse events. JW and AJ reviewed the ulcer photographs. VP drafted the statistical analysis plan, which was signed off by the trial steering committee. VP prepared blinded reports for the data safety monitoring board, conducted all the analyses, and prepared the statistical report. The trial steering committee reviewed the statistical report and agreed to an interpretation of the data. AJ drafted the paper for review by the trial steering committee and CW. All trial steering committee members had access to the statistical analysis. All authors commented on and edited the paper, and approved the final draft for submission.

Funding This trial was funded by the Health Research Council of New Zealand (Reference 16/135). The Health Research Council is a government funded statutory body responsible for managing investment in health research through a competitive peer-review process. The keratin dressing was supplied without charge by Keraplast Technologies. Neither the Health Research Council nor Keraplast Technologies had any role in the conduct and analysis of the trial, nor in the preparation of this paper.

Competing interests None declared.

Patient and public involvement Patients and/or the public were not involved in the design, or conduct, or reporting, or dissemination plans of this research.

Patient consent for publication Not required.

Ethics approval Ethical approval for the trial was obtained from the Northern A Health \& Disability Ethics Committee (16/NTA/142) for the New Zealand study centres and from the institutional ethics committees for the Australian study centres (HREC/17/Alfred/183 and SSA/18/Austin/49).

Provenance and peer review Not commissioned; externally peer reviewed.

Data availability statement Data are available on reasonable request. All requests for de-identified individual participant data or study documents will be considered where the proposed use aligns with public good purposes, does not conflict with other requests or planned use by the trial steering committee, and the requestor is willing to sign a data access agreement. Contact is through the corresponding author.

Open access This is an open access article distributed in accordance with the Creative Commons Attribution Non Commercial (CC BY-NC 4.0) license, which permits others to distribute, remix, adapt, build upon this work non-commercially, and license their derivative works on different terms, provided the original work is properly cited, appropriate credit is given, any changes made indicated, and the use is non-commercial. See: http://creativecommons.org/licenses/by-nc/4.0/.
ORCID iDs

Andrew Jull http://orcid.org/0000-0002-8484-3771

Carolina Weller http://orcid.org/0000-0002-8016-1060

\section{REFERENCES}

1 Morasso MI, Tomic-Canic M. Epidermal stem cells: the cradle of epidermal determination, differentiation and wound healing. Biol Cell 2005;97:173-83.

2 Pastar I, Stojadinovic O, Yin NC, et al. Epithelialization in wound healing: a comprehensive review. Adv Wound Care 2014;3:445-64.

3 Charles CA, Tomic-Canic M, Vincek V, et al. A gene signature of nonhealing venous ulcers: potential diagnostic markers. J Am Acad Dermatol 2008;59:758-71.

4 Tang L, Sierra JO, Kelly R, et al. Wool-derived keratin stimulates human keratinocyte migration and types IV and VII collagen expression. Exp Dermatol 2012;21:458-60.

5 Pechter PM, Gil J, Valdes J, et al. Keratin dressings speed epithelialization of deep partial-thickness wounds. Wound Repair Regen 2012;20:236-42.

6 Margolis DJ, Berlin JA, Strom BL. Which venous leg ulcers will heal with limb compression bandages? Am J Med 2000;109:15-19.

7 Dumville JC, Worthy G, Bland JM, et al. Larval therapy for leg ulcers (Venus II): randomised controlled trial. BMJ 2009;338:b773.

8 Watson JM, Kang'ombe AR, Soares MO, et al. Venus III: a randomised controlled trial of therapeutic ultrasound in the management of venous leg ulcers. Health Technol Assess 2011;15.

9 Iglesias C, Nelson E, Cullum N, et al. Venus I: a randomised controlled trial of two types of bandage for treating venous leg ulcers. Health Technol Assess 2004;8:1-105.

10 Ashby RL, Gabe R, Ali S, et al. VenUS IV (Venous leg Ulcer Study IV) - compression hosiery compared with compression bandaging in the treatment of venous leg ulcers: a randomised controlled trial, mixedtreatment comparison and decision-analytic model. Health Technol Assess 2014;18:1-294.

11 Jull A, Wadham A, Bullen C, et al. Low dose aspirin as adjuvant treatment for venous leg ulceration: pragmatic, randomised, double blind, placebo controlled trial (Aspirin4VLU). BMJ 2017;358:j5157.

12 Jull A, Wadham A, Bullen C, et al. Wool-derived keratin dressings versus usual care dressings for treatment of slow-healing venous leg ulceration: study protocol for a randomised controlled trial (Keratin4VLU). BMJ Open 2018;8:e020319.

13 International Wound Infection Institute. Wound infection in clinical practice: wounds international, 2016.

14 Stacey MC, Burnand KG, Layer GT, et al. Measurement of the healing of venous ulcers. Aust N Z J Surg 1991;61:844-8.

15 Fu X, Kelly R, Marsh C, et al. A concurrent cohort clinical study of functional keratin dressings for treatment of chronic venous leg ulcers. Symposium on advanced wound care. Denver, Colorado, 2013.

16 Vivas AC, Maderal AD, Than MP, et al. Designing clinical trials to bring wound products to market. Int Wound J 2013;10:114-5.

17 Davidson A, Jina NH, Marsh C, et al. Do functional keratin dressings accelerate epithelialization in human partial thickness wounds? A randomized controlled trial on skin graft donor sites. Eplasty 2013;13:e45.

18 Batzer AT, Marsh C, Kirsner RS. The use of keratin-based wound products on refractory wounds. Int Wound J 2016;13:110-5.

19 Nelson EA, Iglesias CP, Cullum N, et al. Randomized clinical trial of four-layer and short-stretch compression bandages for venous leg ulcers (VenUS I). Br J Surg 2004;91:1292-9.

20 Jull A, Walker N, Parag V, et al. Randomized clinical trial of honey-impregnated dressings for venous leg ulcers. Br J Surg 2008;95:175-82.

21 Serena T, Robson MC, Cooper DM, et al. Lack of reliability of clinical/visual assessment of chronic wound infection: the incidence of biopsy-proven infection in venous leg ulcers. Wounds 2006;18:197-202.

22 Food and Drug Administration. Guidance for industry chronic cutaneous ulcer and burn wounds - developing products for treatment. Rockville, Maryland: Food and Drug Administration, 2006.

23 Jull A, Parag V, Walker N, et al. The PREPARE pilot RCT of homebased progressive resistance exercises for venous leg ulcers. $J$ Wound Care 2009;18:497-503. 